\title{
A Portable MIMO Testbed and Selected Channel Measurements
}

\author{
Paul Goud Jr., Robert Hang, Dmitri Truhachev, and Christian Schlegel \\ Department of Electrical and Computer Engineering, University of Alberta, Edmonton, AB, Canada T6G 2V4
}

Received 30 November 2004; Revised 10 July 2005; Accepted 22 August 2005

\begin{abstract}
A portable $4 \times 4$ multiple-input multiple-output (MIMO) testbed that is based on field programmable gate arrays (FPGAs) and which operates in the $902-928 \mathrm{MHz}$ industrial, scientific, and medical (ISM) band has been developed by the High Capacity Digital Communications (HCDC) Laboratory at the University of Alberta. We present a description of the HCDC testbed along with MIMO channel capacities that were derived from measurements taken with the HCDC testbed for three special locations: a narrow corridor, an athletics field that is surrounded by a metal fence, and a parkade. These locations are special because the channel capacities are different from what is expected for a typical indoor or outdoor channel. For two of the cases, a ray-tracing analysis has been performed and the simulated channel capacity values closely match the values calculated from the measured data. A ray-tracing analysis, however, requires accurate geometrical measurements and sophisticated modeling for each specific location. A MIMO testbed is ideal for quickly obtaining accurate channel capacity information.
\end{abstract}

Copyright () 2006 Hindawi Publishing Corporation. All rights reserved.

\section{INTRODUCTION}

Multiple-input multiple-output (MIMO) wireless technology, with its promise to increase channel capacities, is now being considered for use in commercial systems. For example, there have been many proposals to include MIMO technology in the upcoming $802.11 \mathrm{n}$ standard for wireless local area networks (WLAN) [1]. The IEEE 802.11n task group was created to make specifications for WLAN systems (e.g., home theater systems, wireless video services) that achieve a much higher transmission rate than what is currently possible with the $802.11 \mathrm{a} / \mathrm{g}$ standards. The goal for the next generation WLAN standard is a data throughput between 100 and $200 \mathrm{Mb} / \mathrm{s}$. The term MIMO generically means multiple-input multiple-output, however, in this paper we use it synonymously for a wireless channel with multiple inputs/outputs, that is, a multiple antenna channel.

The successful deployment of commercial MIMO systems will require a solid understanding of the channel conditions. There have been many wireless channel models developed that emulate propagation conditions and can be used to provide estimates of MIMO channel capacity. For example, a simple model that is frequently used in simulation studies of Rayleigh fading conditions uses independent identically distributed (i.i.d.) Gaussian random generators to derive the value for each element of a MIMO channel gain matrix $[2,3]$. More sophisticated wireless channel models attempt to account for multiple scatterers and their locations $[4,5]$. Despite their complexity, even these more sophisticated models make many assumptions and ignore common propagation effects such as refraction, diffraction, and reflection loss, or correlations among the different antenna elements. The many assumptions inherent in these models can result in MIMO channel capacity estimates for a location that have large error. The most accurate method to determine the capacity of a MIMO system at a given site is through an analysis of channel measurements.

The collection of the measurements mandates the use of a measurement apparatus (also called a testbed) that can accurately measure the relative gains and phases for all the elements in a MIMO channel gain matrix. In this article, we profile several locations where the MIMO channel capacities we have measured with our testbed are different from what would be expected for general indoor or outdoor channels. In order to explain the discrepancies, we analyze the locations and in some cases perform a detailed ray-tracing analysis.

This paper is organized as follows. In Section 2, we describe several MIMO testbeds that have been developed by other research teams. Section 3 is a review of the basics MIMO channel communications. Our own MIMO testbed design is presented in Section 4. Channel measurements for some interesting locations are given in Section 5 and thoroughly examined. Finally, Section 6 provides a conclusion. 
TABLE 1: Comparison of testbed features.

\begin{tabular}{|c|c|c|c|c|c|}
\hline & $\begin{array}{c}\text { Timing } \\
\text { recovery }\end{array}$ & $\begin{array}{l}\text { Real-time } \\
\text { operation }\end{array}$ & Portability & Size & $\begin{array}{c}\text { Frequency } \\
\text { of operation }\end{array}$ \\
\hline Brigham Young University & With cable & Yes & Limited & $4 \times 4$ & $2.45 \mathrm{GHz}$ \\
\hline Rice University & Receiver loop & Yes & Possible & $2 \times 2$ & $2.4 \mathrm{GHz}$ \\
\hline University of Bristol & Offline & No & Possible & $4 \times 4$ & $5.2 \mathrm{GHz}$ \\
\hline University of Alberta & Receiver loop & Yes & Yes & $4 \times 4$ & $905-925 \mathrm{MHz}$ \\
\hline
\end{tabular}

\section{BACKGROUND}

In addition to the MIMO testbed that has been developed at the University of Alberta and is described later in this paper, several other research teams have developed similar testbeds. We will briefly describe the design and unique features of some of them.

A research team at Brigham Young University has developed a $4 \times 4$ MIMO prototyping testbed that operates at $2.45 \mathrm{GHz}[6]$. Both the transmitter and receiver stations are based on fixed point digital signal processing (DSP) microprocessor development boards and use custom four-channel radio frequency (RF) modules. A computer at the transmitter station generates the four data streams and passes the sampled signals to the DSP board. Each DSP processor pulseshape filters each component of the complex signal and sends the baseband signal to a digital upconverter. At the receiver station, each DSP processor performs matched filtering and passes the filtered outputs to a computer. The computer at the receiver station estimates the transmitted data symbols by deriving an estimate of the channel gain matrix, inverting the channel gain matrix, and multiplying the received samples by the inverted channel gain matrix. System synchronization signal is obtained through a $10 \mathrm{MHz}$ reference signal that passes from the transmitter to the receiver station through a cable.

Another MIMO testbed, developed at Rice University in Houston, Texas [7], operates at $2.4 \mathrm{GHz}$. This $2 \times 2$ testbed is similar to our testbed in that its hardware is based on a field progammable gate array (FPGA) development board. Each FPGA board has two digital-to-analog converters (DACs) and two analog-to-digital converters (ADCs). Off-the-shelf $\mathrm{RF}$ up/downconverter boards from national instruments are also used. A novel feature of the Rice University testbed is its ability to incorporate commercial RF channel emulators. Each emulator can model fading channels such as Rayleigh, Ricean, and Nakagami.

A third testbed of interest is the $4 \times 4$ turbo MIMOOFDM system that was built at the University of Bristol [8]. This system operates at $5 \mathrm{GHz}$ and uses a DSP microprocessor development board for the baseband processing. Timing recovery and channel state information are obtained at the receiver through the use of time-multiplexed preambles that start every frame of data. Each transmitter has a preamble that is orthogonal to all others and has an exclusive timing slot in which to transmit a reference signal. At the receiver, the signal from each receiver antenna is processed by an autocorrelation routine. This routine determines the peak autocorrelation timing for each preamble and uses the information it obtains to calculate the channel state information.

The main features of the three testbeds presented in this section and the HCDC MIMO testbed are compared in Table 1. The testbed of Brigham Young University can operate at limited distances only because of the cable used for synchronization. The testbed of University of Bristol does not allow real-time measurements since the synchronization is done offline. The HCDC testbed and that of Rice University allow for a variety of MIMO channel measurements due to the real-time receiver synchronization loop. Real-time measurement setups give a possibility to track time-varying channels and simplify the selection of interesting measurement locations.

\section{THE MULTIANTENNA MIMO CHANNEL}

A MIMO transmission system uses $N_{t}$ transmit and $N_{r}$ receive antennas. Each antenna $i$ transmits discrete symbols from a complex symbol alphabet each with energy $E_{s i}$ per signaling interval, such that $\sum_{i} E_{s i}=E_{s}$ is constant for each use of the channel. These transmit symbols are modulated by a suitable pulse waveform, upconverted to the desired transmission band, and sent over the $N_{t}$ transmit antennas. The signals from the receive antennas are mixed down to baseband, sampled, and fed into the receiver.

The wireless transmission channel is a linear channel to a high degree of accuracy, and, provided that timing recovery can be accomplished, the received sampled complex signal $y_{i l}$ consisting of an inphase and a quadrature component for the $i$ th receive antenna at time $l$ is given by

$$
y_{i l}=\sum_{j=1}^{N_{t}} \sqrt{E_{s j}} h_{i j} c_{j l}+\eta_{i l},
$$

where $\eta_{i l}$ is a sample of circularly symmetrical complex Gaussian noise with variance $N_{0}, c_{j l}$ is the sampled transmitted signal, and $h_{i j}$ is the complex path gain from transmit antenna $j$ to receive antenna $i$. It contains all linear effects on the signal, such as propagation power loss and phase shifts, fading due to multipath, crosstalk, antenna coupling, and polarization. This model furthermore assumes that the symbol rate is low enough such that frequency selectivity caused by time-of-arrival differences between various multipath replicas of the received signal is not an issue that manifests itself noticeably. This implies symbol rates of about 1 Mbaud or less for indoor transmission, and about 50 kbaud or less for 
outdoor situations [9] which is the case for our system (see Section 4).

The entire MIMO channel can now succinctly be characterized by the linear algebraic relationship

$$
\mathbf{y}=\mathbf{H A c}+\mathbf{n}, \quad \mathbf{A}=\left[\begin{array}{llll}
\sqrt{E_{s 1}} & & & \\
& \sqrt{E_{s 2}} & & \\
& & \ddots & \\
& & & \sqrt{E_{s N_{t}}}
\end{array}\right],
$$

where $\mathbf{H}$ is an $N_{r} \times N_{t}$ rectangular matrix of channel gains $h_{i j}$ and $\mathbf{c}$ is a vector of $N_{t}$ transmitted symbols $c_{j l}$. The information theoretic capacity of the discrete channel in (2) can be calculated from basic information theoretic concepts [10] as

$$
C_{I}=\log _{2} \operatorname{det}\left(\mathbf{I}+\frac{\rho}{N_{t}} \mathbf{H E H}^{+}\right)[\text {bits/channel use }]
$$

where $\rho=E_{s} / N_{0}$ is the signal-to-noise ratio per symbol,

$$
\mathbf{E}=\frac{1}{E_{s}}\left[\begin{array}{cccc}
E_{s 1} & & & \\
& E_{s 2} & & \\
& & \ddots & \\
& & & E_{s N_{t}}
\end{array}\right]
$$

and $\mathbf{H}^{+}$is the conjugate transpose of $\mathbf{H}$. Since the channel parameters are time varying, $C_{I}$ is interpreted as the "instantaneous" channel capacity for a given channel realization $\mathbf{H}$. For a time-varying channel this capacity has to be averaged over all realizations of the MIMO channel matrix $\mathbf{H}$ to calculate the ergodic channel capacity $C=E_{\mathbf{H}}\left(C_{I}\right)$. Telatar [11] has presented closed form solutions for $C$ in the case where the $h_{i j}$ are independent, equal-variance complex Gaussian fading channel gains.

The matrix $\mathbf{H}$ can be decomposed using the singular value decomposition (SVD) [12] $\mathbf{H}=\mathbf{U D V}^{+}$where $\mathbf{U}$ and $\mathbf{V}$ are unitary matrices, and the matrix $\mathbf{D}$ contains the singular values $\left\{d_{n}\right\}$ of $\mathbf{H}$ on its diagonal, which are the positive square roots of the nonzero eigenvalues of $\mathbf{H H}^{+}$or $\mathbf{H}^{+} \mathbf{H}$. This allows the instantaneous capacity to be written in terms of the singular values as

$$
C_{I}=\sum_{n=1}^{N} \log _{2}\left(1+\frac{d_{n}^{2} E_{n}}{N_{0}}\right) \longrightarrow C_{W}=\sum_{n=1}^{N} \log _{2}\left(\frac{d_{n}^{2} \mu}{N_{0}}\right)
$$

and the maximizing energy levels for each subchannel are found via the the well-known water-filling theorem [13] as

$$
\begin{aligned}
& E_{n}=\mu-\frac{N_{0}}{d_{n}^{2}}, \quad \frac{N_{0}}{d_{n}^{2}}<\mu, \\
& E_{n}=0, \quad \frac{N_{0}}{d_{n}^{2}} \geq \mu
\end{aligned}
$$

leading to the water-filling capacity $C_{W}$ in (5). $\mu$ is the waterfilling level chosen such that $\sum_{n} E_{n}=E_{s}$.

However, if channel knowledge is not available at the transmitter uniformly distributing the energy over all component channels, using $E_{n}=E_{s} / N_{t}$, maximizes capacity. This special case is known as the symmetric capacity. Fundamentally, the capacity of a MIMO channel is governed by the singular values of $\mathbf{H}$ which determine the channel gains of the independent equivalent parallel channels resulting from the SVD.

Let us consider normalized matrix of path channel gains $\widetilde{\mathbf{H}}=1 / \alpha \mathbf{H}$ where

$$
\alpha=\sqrt{\frac{\left\|\mathbf{H H}^{+}\right\|}{N_{t} N_{r}}}
$$

is the channel attenuation coefficient. If the channel paths $\tilde{h}_{i j}$ are uncorrelated, as happens when there is a multitude of scatterers that reflect the radio waves between transmitters and receiver, a typical observed channel realization will be of high rank with eigenvalues of $\tilde{\mathbf{H}} \tilde{\mathbf{H}}^{+}$distributed according to a Wishart distribution [11]. In this case the MIMO capacity will grow nearly linearly with the number of inputs and outputs, that is, if we let $N=\min \left(N_{r}, N_{t}\right)$, then $C_{I}=O(N)$. If, however, the component channels show strong correlation, such as occurs in scatter-free long-distance wireless connections, for example, in a satellite-ground radio link, or approximately in the green field and narrow corridor measurements discussed below, the rows $\tilde{\mathbf{h}}_{j}$ of $\tilde{\mathbf{H}}$, the array response vectors, will become approximately equal and equal to all-ones vectors $(11 \cdots 1)$ due to normalization. The matrix $\tilde{\mathbf{H}}$ becomes approximately equal to an $N_{r} \times N_{t}$ matrix of ones which has only one nonzero singular value, $d=\sqrt{N_{t} N_{r}}$. As a result

$$
C_{\mathrm{low}} \approx \log _{2}\left(1+\alpha^{2} \rho N_{r}\right) .
$$

In this case the channel capacity grows only logarithmically with the number of (receive) antennas, and the system realizes only the power gain provided by having a number of virtual receive antenna, and not the diversity gain realized by a high-rank channel.

Real-world situation will lie somewhere between these two extremes, with the capacity determined by the complex propagation environment in which the system has to function. This leads to the necessity of carefully analyzing and measuring such candidate environments to obtain precise channel coefficients.

\section{TESTBED DESCRIPTION}

The iCORE HCDC Lab has developed a flexible $4 \times 4$ MIMO testbed that allows real-time characterization of MIMO wireless channels in a flat-fading environment. The testbed determines the coefficients of the $4 \times 4$ MIMO transmission matrix. The MIMO testbed consists of an independent transmitter and receiver that operate in the 902-928 MHz ISM band. Battery and voltage regulation circuits have been developed for both stations which means that testbed usage is not restricted to locations near electrical power receptacles.

Figure 1 shows the MIMO transmitter. From left to right, it consists of a GVA290 development board (manufactured by GV and Associates Inc.), inline filters, a four-channel upconverter module (from SignalCraft Technologies Inc.), and a multiantenna structure. The multiantenna structure creates 


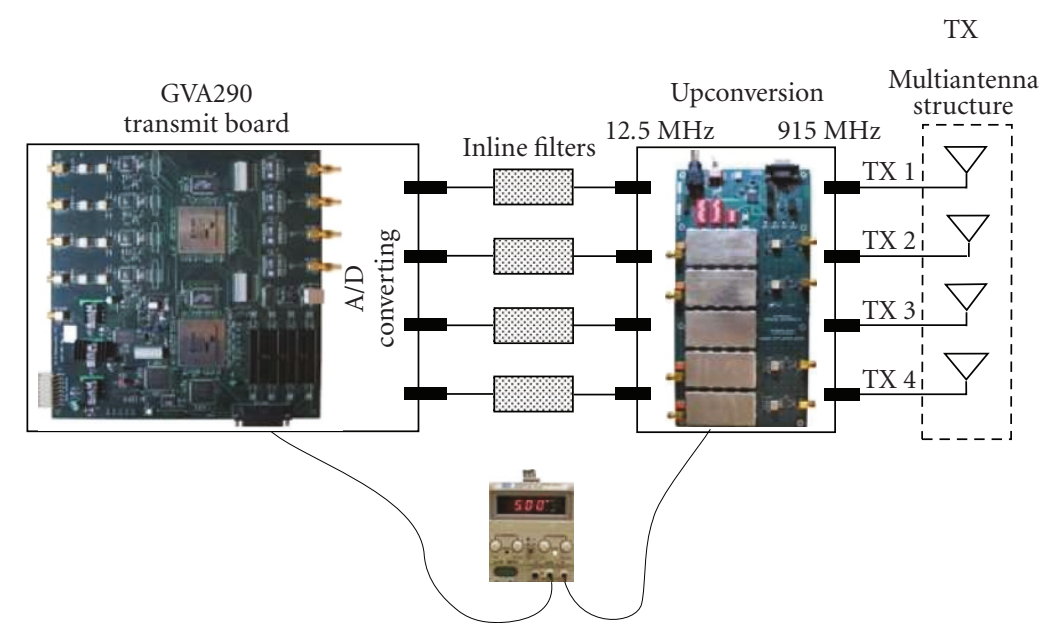

FIGURE 1: MIMO testbed transmitter.

RX

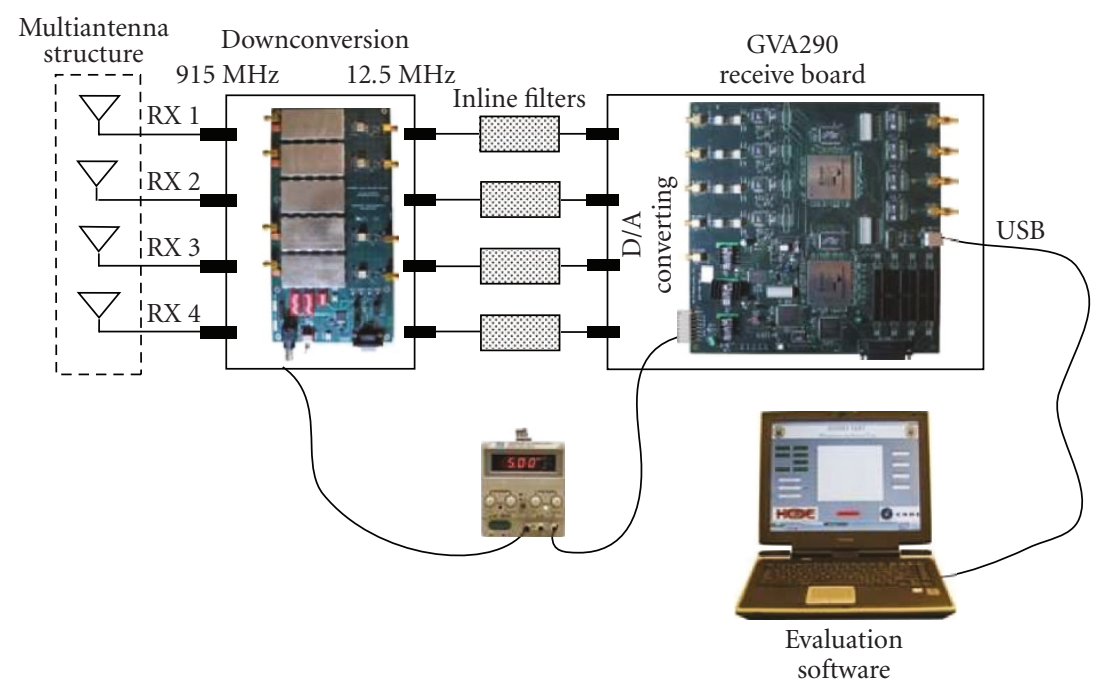

FIgURE 2: MIMO testbed receiver.

a set of four dipole antennas with adjustable antenna spacing through the use of magnet-mounted monopole antennas attached to an iron sheet. The GVA290 board is populated with two Xilinx Virtex-E 2000 FPGAs, four 12-bit Analog Devices AD9762 digital-to-analog converters (DACs), and four 12-bit Analog Devices AD9432 analog-to-digital converters (ADCs). One FPGA, clocked at $50 \mathrm{MHz}$, creates four Walsh codes of length 32 (each code is overlaid with an m-sequence to improve the spectral characteristics), one for each of the independent paths of the $4 \times 4$ MIMO channel measurement testbed. Each code is continuously repeated at a rate of $15.625 \mathrm{kHz}$. Therefore, the chip rate of each channel is $500 \mathrm{kchips} / \mathrm{s}$ and a chip period corresponds to a propagation distance of $600 \mathrm{~m}$. The chipping rate is low enough that we can safely assume that the channel is not frequency selective in any indoor environment or in outdoor environments where buildings are in close proximity. A raised-cosine pulse, with a roll-off factor of 0.31 , is used to shape the four baseband signals before digital upconversion to an intermediate frequency (IF) of $12.5 \mathrm{MHz}$ occurs. The four IF signal sample streams exit the FPGA and are converted to analog waveforms by the DACs of the GVA290 board which are also clocked at $50 \mathrm{MHz}$. The outputs of the DACs are connected to the SignalCraft module through inline low-pass filters with a cutoff frequency of $15 \mathrm{MHz}$. The RF board then upconverts these four independent IF waveforms $\left(\mathrm{TX}_{i}, 1 \leq\right.$ $i \leq 4)$ to the $902-928 \mathrm{MHz}$ band for transmission over the air through the "multiantenna structure."

Figure 2 shows the MIMO receiver. From left to right, it consists of the same multiantenna structure as used by the transmitter: an RF downconverter board (manufactured by SignalCraft Technologies Inc.) with four independent receive 


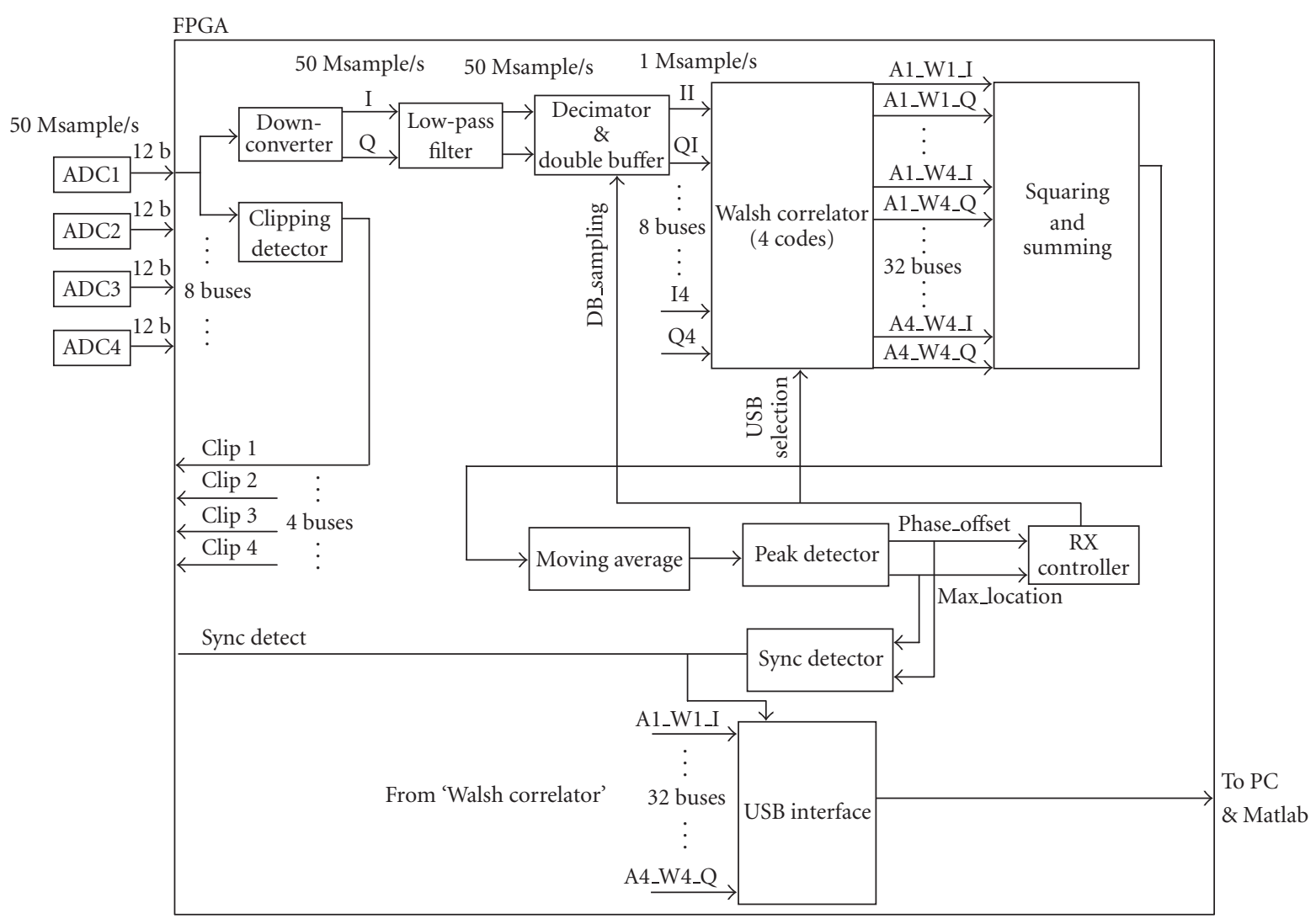

Figure 3: Receiver FPGA architecture.

paths, inline filters, and a GVA290 board. Each of the receive paths $\left(\mathrm{RX}_{i}, 1 \leq i \leq 4\right)$ is downconverted from the ISM RF band to an IF of $12.5 \mathrm{MHz}$ by the RF module. The four receive passband signals are then sampled by the ADCs of the GVA290 board. The four sample streams $\left(\mathrm{ADC}_{i}, 1 \leq i \leq 4\right)$ are processed by the FPGAs at a clock rate of $50 \mathrm{MHz}$.

Figure 3 shows the architecture of the receiver implemented within the FPGA. The samples of the incoming passband signals are quantized with 12 bits of accuracy. A clipping detector circuit operates on each of the ADC signals and notifies the operator if an incoming signal exceeds the dynamic range of the ADCs. Then, for each of the four datapaths, the samples are digitally downconverted to an inphase (I) and a quadrature (Q) component. The low-pass filter, a simple finite-impulse response (FIR) filter with five coefficients and a cutoff frequency of $1 \mathrm{MHz}$, ensures that no aliasing occurs after decimation. Following the filter is the "decimator and double buffer" block which performs the decimation from $50 \mathrm{MHz}$ to $1 \mathrm{MHz}$. There is a control signal coming from the RX controller (described later) that controls the decimation instant such that the signal is sampled as close as possible to the ideal sampling instant of the received raisedcosine pulse. The double buffer has two buffers that are filled alternatively. While one buffer is being filled with the samples for a period of a Walsh code, the other buffer is read out and its content is processed by the following block, the Walsh correlator. This allows for block processing, where one block is being received, while a previous block is being processed.

The Walsh correlator block performs the code-matched filtering. The data from ADC1 will be correlated with Walsh code 1 leading to the "A1_W1_I" and "A1_W1_Q" buses, Walsh code 2 leading to "A1_W2_I" and "A1_W2_Q," up to Walsh code 4 ("A1_W4_I" and "A1_W4_Q"). The same applies to the other ADCs resulting in 16 pairs of signals that are represented by "Ai_Wj_I" and "Ai_Wj_Q" for $\mathrm{i}$ and $\mathrm{j}$ ranging from 1 to 4 in Figure 3. The result of the code-matched filtering is then noncoherently combined by the squaring and summing block to avoid phase recovery. In order to make the synchronization algorithm more robust to noise, a running moving average is applied to the output of the squaring and summing block. In the moving average, the incoming sample is added to the previous output of the moving average multiplied by a forgetting factor, a real number strictly less than unity but close to unity. The effect of this moving average is to raise the signal to noise ratio of the signal. This reliable output is then used by the early-late gate peak detector [14]. The peak detector will tell the RX_controller the sample that contains the maximum of the code-matched filtering operation via the "max_location" signal. The "phase_offset" signal tells the RX_controller how far away the sample is from the ideal sampling point of the raised-cosine pulse. The RX_controller uses that information to move the sampling instant of the 
decimator and double buffer block with the DB_sampling signal. This feedback loop is constantly running to adjust code synchronization. The sync detector is a block that detects if the receiver has locked on to the incoming signal. Once synchronization has been established, the result of the Walsh correlator block can be uploaded to the PC connected to the FPGA board via the USB interface. The correct samples are selected by the RX_controller block via the USB_selection signal. These complex samples represent the channel gains of the $4 \times 4 \mathrm{MIMO}$ channel matrix. They are processed by the software Matlab running on the PC to obtain the instantaneous channel capacity. The synchronization scheme explained above is further described and its performance analysis is shown in [15].

Our MIMO receiver performs the measurements noncoherently and there are two reasons why this is possible. First of all, the maximum frequency error between the two stations, which is defined by the error in the clock signals used at each station, is much less than the inverse of the period of the spread spectrum signal:

$$
\Delta f<\frac{1}{T_{s}} .
$$

This means that the phase shift will be practically a complex constant for each correlation that occurs in the Walsh correlator. Since we later square the correlation values, the phase shift has no impact. Secondly, the phase difference between the transmitter and receiver stations can be factored out of the channel capacity equation. In both the transmitter and receiver, all four channels use the same oscillator, thus, the phase difference will be the same for all four channels. If we let $\phi$ represent the complex phase difference value, our equation for the received signal vector becomes

$$
y=\phi \mathbf{H x}
$$

and our capacity equation becomes

$$
C_{I}=\log _{2} \operatorname{det}\left(\mathbf{I}+\frac{\rho}{N_{t}} \phi \phi^{+} \mathbf{H E H}^{+}\right)[\text {bits/channel use }]
$$

and the $\phi \phi^{+}$product is 1 .

The $902-928 \mathrm{MHz}$ ISM band (also denoted by $915 \mathrm{MHz}$ band) was chosen for our measurement campaigns because it is unlicensed and has no interfering cellular or wireless LAN signals. Moreover, the components for the RF module are widely available, cheap, and easy to design with. Because of the testbed's modular design, it is straightforward to change the RF boards of the transmitter and receiver to measure a different frequency such as the unlicensed 2.4 GHz ISM band or the unlicensed $5 \mathrm{GHz}$.

\section{CHANNEL MEASUREMENTS FOR SELECT CHANNELS}

In this section, we present a select number of unusual channel situations with their MIMO measurements. In some cases, we offer simple analytical models which capture the essence of the MIMO channel as it pertains to its information theoretic capacity. Many of the measurements are available to other research teams to download from our MIMO website (http://www.ece.ualberta.ca/ mimo). In particular, we will present three locations we found to be of interest: a narrow corridor, an open field with a nearby chain fence, and a parkade [16]. A signal-to-noise ratio (SNR) of $20 \mathrm{~dB}$ was used for all our channel capacity calcuations since this is a typical indoor value.

\subsection{Narrow corridor}

A narrow corridor is an intriguing location for making MIMO channel measurements because of its tendency to act like a waveguide and increase the correlation between the signals at the receiver antennas. A previous corridor study [17] of MIMO channel capacity at $1.95 \mathrm{GHz}$ found that channel capacity decreased with distance down the hall. The authors of that paper believe that this decrease is due to the keyhole effect. This behavior is different from the rich multipath environment that is typical of indoor offices even though corridors are commonly found in office settings.

Our investigation of MIMO channel capacity in a narrow corridor occurred in the northern corridor on the 5 th floor of the Civil/Electrical Engineering Building at the University of Alberta campus. The corridor has the dimensions of $2.65 \mathrm{~m}$ wide by $2.5 \mathrm{~m}$ in height. It has walls constructed of concrete blocks and a suspended ceiling. The map in Figure 4 shows the transmitter and receiver locations. The transmitter was placed at one end of the hall (location TX) and the receiver station was put at three different locations: L1 (8 meters), L2 (20 meters), and L3 (35 meters). The line-of-sight path is marked by letter B. An analysis of our measurement campaign data confirms the findings of the previous study. The MIMO channel capacities were calculated from the measured transmission matrices using (3). Table 2 shows that the channel capacity drops as the receiver cart is moved down the hall. Figure 5 shows plots of the cumulative distribution functions of the capacities for the three locations.

Figure 6 gives an intuitive understanding of what occurs. Radio waves that strike the concrete walls at a small angle of incidence $\theta$ (ray A) will require many reflections to reach the receiver. Since power is lost with each reflection, multireflected rays will be heavily attenuated at the end of the hall. Those waves that strike a wall with a glancing blow (ray C) will require fewer reflections to reach the receiver and thus suffer less attenuation. In addition to this, studies [18] of the $\mathrm{RF}$ reflection properties of concrete blocks have shown that smaller angles of incidence have lower power reflection coefficients. Therefore, multibounce rays are additionally attenuated by having a lower reflection coefficient with every reflection. These effects explain why propagation along a narrow corridor should be very effective in eliminating multipath components and reducing the MIMO channel rank.

The greatly diminished multipath propagation environment makes it easy to perform a ray-tracing analysis of the site. The reflection coefficient for a radio signal off a plane surface can be calculated when five values are known: the 
TABle 2: Capacity in the corridor.

\begin{tabular}{lccc}
\hline & $\begin{array}{c}\text { Station } \\
\text { separation } \\
\text { (meters) }\end{array}$ & $\begin{array}{c}\text { Average channel } \\
\text { capacity from measurements } \\
\text { (bits/use) }\end{array}$ & $\begin{array}{c}\text { Channel capacity } \\
\text { from the model } \\
\text { (bits/use) }\end{array}$ \\
\hline Location 1 & 8 & 19.226 & 20.720 \\
Location 2 & 20 & 12.270 & 11.187 \\
Location 3 & 35 & 12.180 & 10.226 \\
\hline
\end{tabular}

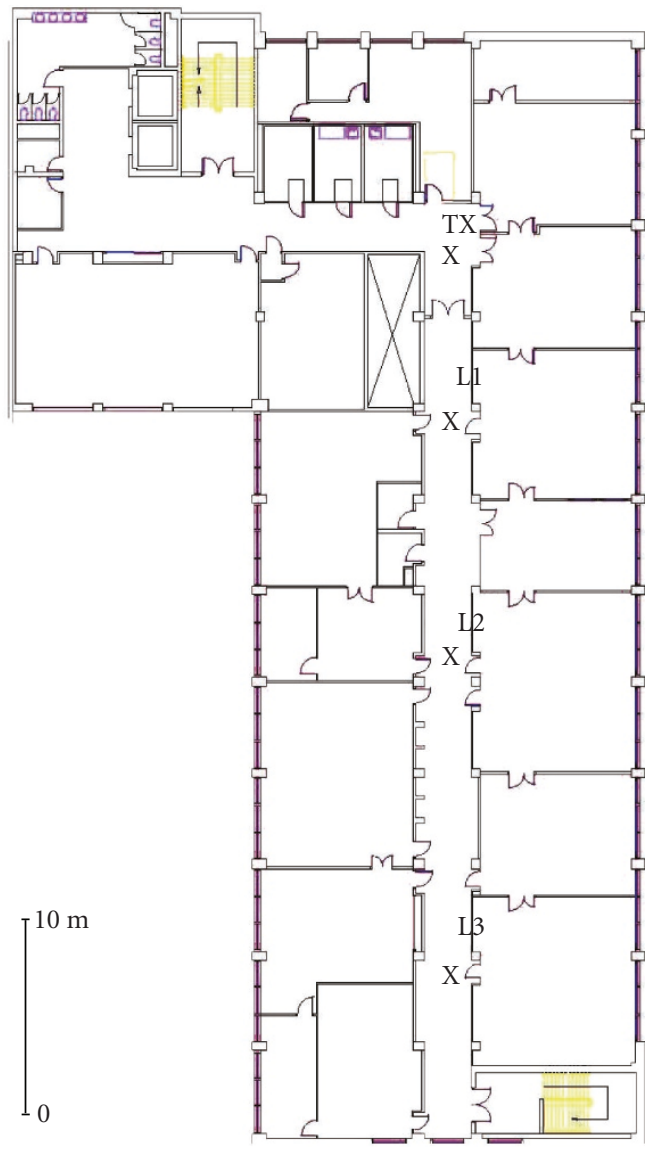

FIgURe 4: Corridor map.

wavelength, the relative dielectric constant of the material, the conductivity of the material, the polarization of the radio wave, and the angle of incidence [19]. For concrete, a typical relative dielectric constant is 5 and a typical conductivity is $0.001 \mathrm{mho} / \mathrm{m}$.

A Matlab program was written which simulates the lineof-sight (LOS) path, the radiation reflected off the floor, and the rays that are reflected once, twice, and three times off the walls. Since our dipole antennas were vertically polarized, a vertically polarized reflection will occur off the floor and a horizontally polarized reflection will occur off the walls. A 180 degree phase shift will occur for a vertically polarized reflection with a large angle of incidence.

Reflection coefficients were calculated for all the rays for the three locations with our estimates of the incidence angles.

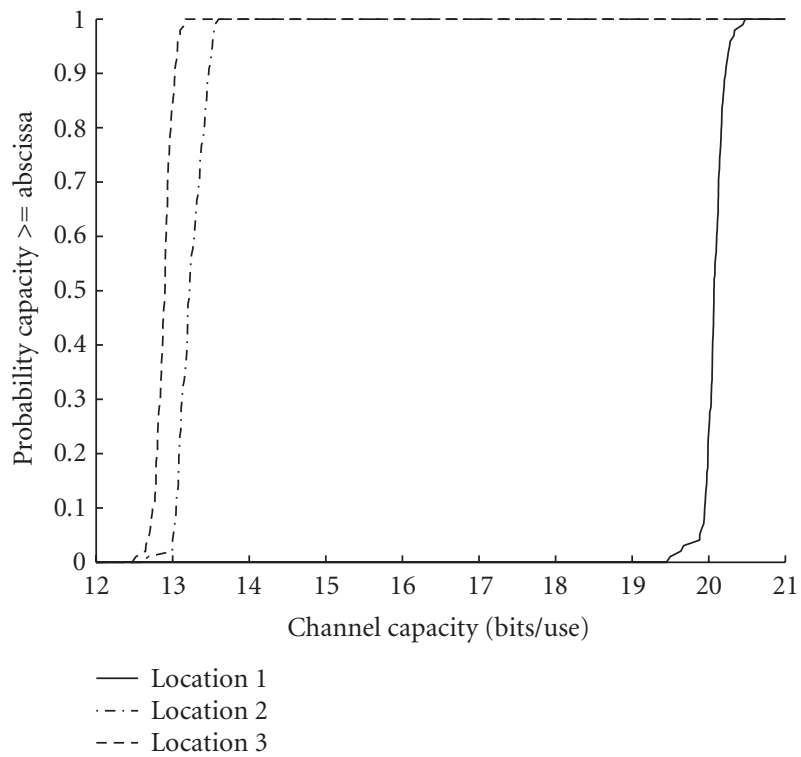

FIGURE 5: Cumulative distribution function of the capacity measurements in the corridor.

These coefficients were used to calculate the complex signals at the receiver. Channel gain matrices were created by adding all the contributions and then the expected MIMO capacity was calculated. The average measured capacity values appear in the third column of Table 2 and the capacities calculated by the program appear in column four. The two sets of numbers match closely.

\subsection{Athletics field}

A second measurement campaign that provided surprising results was the Corbett sports field location at the University of Alberta. Figure 7 is a map of the location. Since this location is an open field, it was our expectation that this would be close to an ideal nonscattering environment and our MIMO channel would have low rank. The closest buildings are 100 meters away and do not have geometrics that would easily lend themselves to reflecting rays back towards the receiver station.

A theoretical analysis of an open field environment [4] predicts that MIMO channel capacity will decrease as the distance between the transmitter and receiver stations increases. The further apart the two stations are, the closer LOS path lengths are to being equal and, hence, the normalized 


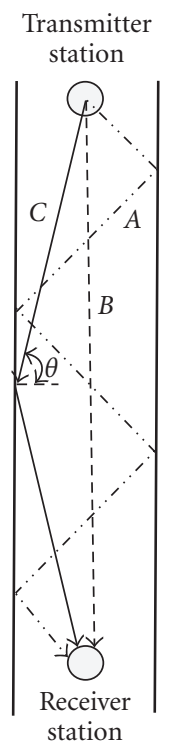

Figure 6: Corridor diagram.

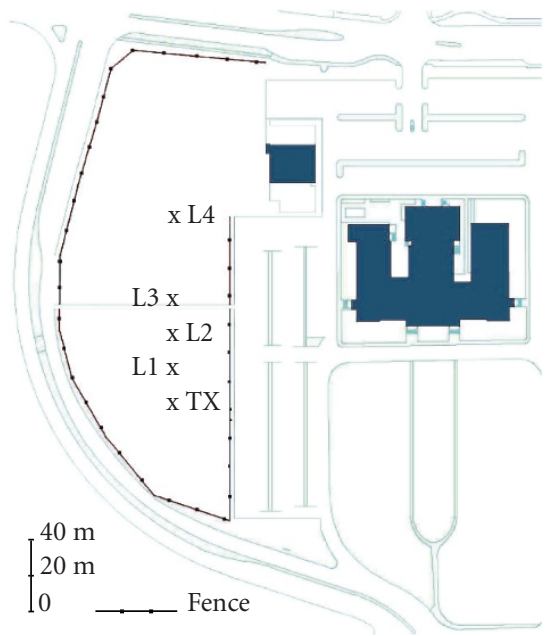

Figure 7: Corbett field map.

channel gain matrix should approach an all-ones matrix which has very low rank. In fact, a measurement campaign performed on an open farm field yielded exactly these results. The same station separations were used for both the farm and sports field locations. The average channel capacities for the farm are shown in the second column of Table 3.

Much to our surprise, the SNR-normalized channel capacity on the sports field actually increased as the station separation increased. Moreover, the values are much higher than we expected. Our investigation into the unexpected results focused on a wire mesh fence that has a height between $2 \mathrm{~m}$ and $4 \mathrm{~m}$, which we had not noticed originally as a significant scatterer, located $25 \mathrm{~m}$ to the right of both the receiver and transmitter stations. It runs in parallel to the LOS between the two stations. To the left of the stations there exists another fence that is curved and is at least $40 \mathrm{~m}$ away.

As was done in the narrow corridor case, a ray-tracing program was written for the location. The channel simulation included the line-of-sight path, the radiation reflected off the ground, and the reflected rays off the two fences. The vertically polarized reflection coefficient for the grassy ground was once again calculated with a typical relative dielectric constant of 10 and conductivity value of $0.005 \mathrm{mho} / \mathrm{m}$.

The different propagation distances were accounted for by including a free space attenuation factor with all the paths [20]. The capacity values from the program appear in the last column of Table 3 . The simulated capacities increase with distance in a similar fashion to our measured values.

\subsection{Parkade}

There are several publications that describe MIMO measurement campaigns for indoor office environments and calculate the channel capacity $[21,22]$. A parkade is different from an indoor office environment in several respects. First, a typical indoor office has building materials (e.g., gyproc, glass, wood) that are not found in a parkade. In addition, an indoor office environment usually has interior walls and doors that are not present in a parkade. We could find no previous published results for a parking lot location.

Level P1 of the underground parkade in the ECERF (Electrical and Computer Engineering Research Facility) building on the University of Alberta campus was selected for a MIMO measurement campaign (see Figure 8). The ECERF parkade is a typical parkade in that it has concrete walls, floors, and pillars. At the time the measurements were taken, many of the parking spots were filled with cars. The map in Figure 9 shows the location of the transmitter station and receiver measurement places.

The channel capacities calculated from our parkade measurements (see Table 4) were slightly lower than what we had measured for indoor office environments (typically about $20 \mathrm{bits} /$ channel use for a $4 \times 4$ system). Thus, the features of an indoor office may be more effective in creating a rich multipath environment than the vehicles present in the parkade. The average channel capacities for locations L1, L2, and L3 are lower than those for locations L4 and L5. This is not surprising since a LOS path exists in the former cases.

\section{CONCLUSION}

In this paper, we have described our portable $4 \times 4 \mathrm{MIMO}$ testbed and presented the measured MIMO capacity for several special locations. The measured MIMO capacities for these locations are different from what would be calculated from general indoor and outdoor wireless propagation models. For two of the locations, the propagation effects are such that an accurate ray-tracing analysis is possible. The channel capacities derived from the analysis are close to our measured values. A ray-tracing analysis, however, can only be used in special cases and requires considerable effort to obtain geometric measurements. 
TABle 3: Capacity in the field.

\begin{tabular}{lcccc}
\hline & $\begin{array}{c}\text { Station separation } \\
\text { (meters) }\end{array}$ & $\begin{array}{c}\text { U of A farm measured average } \\
\text { channel capacity } \\
\text { (bits/use) }\end{array}$ & $\begin{array}{c}\text { Measured Corbett field } \\
\text { average channel capacity } \\
\text { (bits/use) }\end{array}$ & $\begin{array}{c}\text { Field with a fence model } \\
\text { channel capacity } \\
\text { (bits/use) }\end{array}$ \\
\hline Location 1 & 20 & 11.314 & 11.097 & 13.957 \\
Location 2 & 40 & - & 17.097 & 20.016 \\
Location 3 & 60 & 9.383 & 18.079 & 20.535 \\
Location 4 & 100 & 9.860 & 19.439 & 19.051 \\
\hline
\end{tabular}

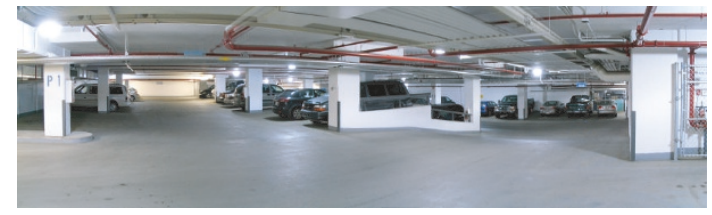

Figure 8: Parkade photo.

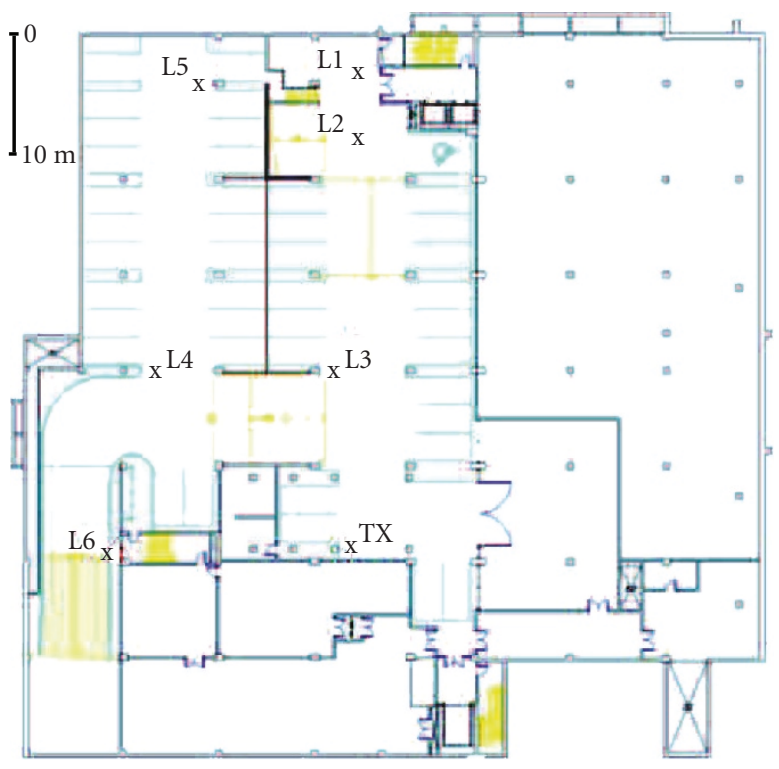

Figure 9: Parkade map.

The benefits of a real-time MIMO testbed are many. It allows real-world characterization of MIMO propagations that are difficult to model. It allows researcher to quickly find channels with interesting characteristics (e.g., outdoor channels with high matrix rank or indoor channel with low matrix rank) in order to study them and gain a better understanding of the advantages and limitations of MIMO communications. Finally, these MIMO channel matrices can be stored and used in link level simulations of communications systems in order to obtain results that are representative of real-world situations.

\section{ACKNOWLEDGMENTS}

This work was supported by the Alberta Informatics Circle of Research Excellence (iCORE), the Alberta Ingenuity Fund,
TABLE 4: Capacity in the parkade.

\begin{tabular}{lccc}
\hline & $\begin{array}{c}\text { Average channel } \\
\text { capacity } \\
\text { (bits/use) }\end{array}$ & $\begin{array}{c}\text { Max. channel } \\
\text { capacity } \\
\text { (bits/use) }\end{array}$ & $\begin{array}{c}\text { Min. channel } \\
\text { capacity } \\
\text { (bits/use) }\end{array}$ \\
\hline Location 1 & 15.972 & 16.865 & 15.287 \\
Location 2 & 17.616 & 17.616 & 16.615 \\
Location 3 & 14.231 & 16.616 & 13.008 \\
Location 4 & 18.463 & 19.632 & 17.127 \\
Location 5 & 18.752 & 19.994 & 17.717 \\
\hline
\end{tabular}

the Natural Sciences and Engineering Research Council (NSERC), the Canadian Foundation for Innovation (CFI), and the National Science Foundation (NSF) of the United States. The authors gratefully acknowledge Ivan Kocev and Tobias Kiefer of the University of Applied Sciences in Offenburg, Germany for their considerable effort in collecting MIMO channel measurements.

\section{REFERENCES}

[1] P. Mannion, "IEEE pushes WLANs to 'nth' degree," Electronic Engineering Times, p. 8, July 2004.

[2] D. Chizhik, F. Rashid-Farrokhi, J. Ling, and A. Lozano, "Effect of antenna separation on the capacity of BLAST in correlated channels," IEEE Communications Letters, vol. 4, no. 11, pp. 337-339, 2000.

[3] G. J. Foschini and M. J. Gans, "On limits of wireless communications in a fading environment when using multiple antennas," Wireless Personal Communications, vol. 6, no. 3, pp. 311-335, 1998.

[4] D. Gesbert, H. Bölcskei, D. A. Gore, and A. J. Paulraj, "Outdoor MIMO wireless channels: models and performance prediction," IEEE Transactions on Communications, vol. 50, no. 12, pp. 1926-1934, 2002.

[5] D.-S. Shiu, G. J. Foschini, M. J. Gans, and J. M. Kahn, "Fading correlation and its effect on the capacity of multielement antenna systems," IEEE Transactions on Communications, vol. 48, no. 3, pp. 502-513, 2000.

[6] J. W. Wallace, B. D. Jeffs, and M. A. Jensen, "A real-time multiple antenna element testbed for MIMO algorithm development and assessment," in Proceedings of IEEE Antennas and Propagation Society International Symposium, vol. 2, pp. 17161719, Monterey, Calif, USA, June 2004.

[7] P. Murphy, F. Lou, A. Sabharwal, and J. P. Frantz, "An FPGA based rapid prototyping platform for MIMO systems," in Proceedings of 37th Asilomar Conference on Signals, Systems and Computers, vol. 1, pp. 900-904, Pacific Grove, Calif, USA, November 2003.

[8] T. Horseman, J. Webber, M. K. Abdul-Aziz, et al., "A testbed for evaluation of innovative turbo MIMO-OFDM 
architectures," in Proceedings of 5th European Personal Mobile Communications Conference (EPMCC '03), pp. 453-457, Glasgow, Scotland, UK, April 2003.

[9] Guidelines for Evaluation of Radio Transmission Technologies for IMT-2000, Recommendation ITU-R M.1225, 1997.

[10] T. M. Cover and J. A. Thomas, Elements of Information Theory, John Wiley \& Sons, New York, NY, USA, 1991.

[11] I. E. Telatar, "Capacity of multi-antenna Gaussian channels," European Transactions on Telecommunications, vol. 10, no. 6, pp. 585-595, 1999.

[12] R. A. Horn and C. R. Johnson, Matrix Analysis, Cambridge University Press, New York, NY, USA, 1990.

[13] R. G. Gallager, Information Theory and Reliable Communication, John Wiley \& Sons, New York, NY, USA, 1968.

[14] J. G. Proakis, Digital Communications, McGraw-Hill, New York, NY, USA, 4th edition, 2001.

[15] R. Hang, C. Schlegel, W. A. Krzymien, and P. Goud Jr., "A robust timing recovery algorithm for spread-spectrum packet radio systems," in Proceedings of 16th International Conference on Wireless Communications (Wireless '04), pp. 446-463, Calgary, Alberta, Canada, July 2004.

[16] I. Kocev and T. Kiefer, "Implementation and capacity potential verification of multiple antenna transmission systems," Master's thesis, University of Applied Sciences Offenburg, Offenburg, Germany, September 2004.

[17] D. Porrat, P. Kyritsi, and D. C. Cox, "MIMO capacity in hallways and adjacent rooms," in Proceedings of IEEE Global Telecommunications Conference (GLOBECOM '02), vol. 2, pp. 1930-1934, Taipei, Taiwan, November 2002.

[18] W. Honcharenko and H. L. Bertoni, "Transmission and reflection characteristics at concrete block walls in the UHF bands proposed for future PCS," IEEE Transactions on Antennas and Propagation, vol. 42, no. 2, pp. 232-239, 1994.

[19] E. C. Jordan and K. G. Balmain, Electromagnetic Waves and Radiating Systems, Prentice-Hall, Englewood Cliffs, NJ, USA, 2nd edition, 1968.

[20] M. Martone, Multiantenna Digital Radio Transmission, Artech House, Norwood, Mass, USA, 1st edition, 2002.

[21] A. L. Swindlehurst, G. German, J. Wallace, and M. Jensen, "Experimental measurements of capacity for MIMO indoor wireless channels," in Proceedings of IEEE 3rd Workshop on Signal Processing Advances in Wireless Communications (SPAWC '01), pp. 30-33, Taoyuan, Taiwan, March 2001.

[22] P. Goud Jr., C. Schlegel, R. Hang, et al., "MIMO channel measurements for an indoor office environment," in Proceedings of IEEE Wireless Conference, pp. 423-427, Calgary, Alberta, Canada, July 2003.

Paul Goud Jr. received the B.S. degree in electrical engineering from the University of Alberta, Canada in 1989 and the M.S. degree in electrical engineering from the University of Calgary, Canada in 1991. His graduate research was conducted at TRLabs's wireless research laboratory. In 1992, Paul joined Glenayre R\&D Inc. as a DSP/Communications Engineer. At Glenayre, he worked on many wireless trans-

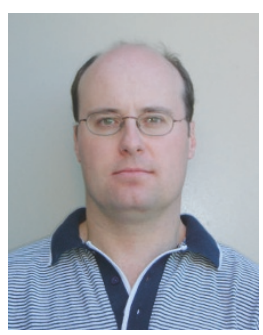
mitter, receiver and handheld device development projects. In 2000, he joined the Wireless Products Division of PMC-Sierra Inc. in Burnaby, BC, and held the positions of Product Validation Engineer and Applications Engineer. Since 2002, Paul has been a Research Engineer in the iCORE High Capacity Digital
Communications (HCDC) Laboratory at the University of Alberta. $\mathrm{He}$ is the coauthor of 4 wireless technology patents and has over 13 years of experience in the design and development of radio transmitters and receivers. His research interests include embedded systems, mobile radio systems, and MIMO technology.

Robert Hang received the "Diplôme d'Ingénieur" (M.Eng.) from ENSEA, Cergy, France, and the M.S. degree from the University of Alberta, Edmonton, AB, Canada, both in electrical engineering, in 1996 and 1998, respectively. In 1999, he joined the Applied Research Department of Bellcore (now Telcordia Technologies), in Red Bank, NJ, USA. While at Bellcore, he worked on a PACS radio port design (PACS is a low-tier TDMA-based cellular system), and on synchronization algorithms for OFDM-based wireless data systems. In 2001, he joined ArrayComm, Freehold, NJ, USA. At ArrayComm, he was involved in the design of user terminals for i-BURST, a high-speed, high-user capacity broadband wireless Internet access system. From January 2003 to July 2005, he was with the High Capacity Digital Communications (HCDC) Laboratory of the University of Alberta. At HCDC, he was responsible for hardware and HDL designs of various projects involving MIMO communications, LDPC decoding, and fast packet synchronization. He joined Cygnus Communications Canada Co. in July 2005 to become the Project Manager for physical layer design of Cygnus 802.16 ASIC. His interests include digital communications and implementation of wireless communications systems.

Dmitri Truhachev was born in Saint Petersburg, Russia, in 1978. He received the B.S. degree in applied mathematics from Saint Petersburg State Electro Engineering University, Saint Petersburg, Russia, in 1999 and the Ph.D. degree in electrical engineering in 2004 from Lund University, Lund, Sweden. In 2004 he joined High Capacity Digital Communications Laboratory at University of Alberta, Edmonton, Canada as a Postdoctoral Fellow. His major research interests include communications, coding theory, and ad-hoc networks.

Christian Schlegel received the Dipl. El. Ing. ETH degree from the Federal Institute of Technology, Zurich, in 1984, and the M.S. and Ph.D. degrees in electrical engineering from the University of Notre Dame, Notre Dame, Ind, in 1986 and 1989. In 2001, he was named iCORE Professor for High-Capacity Digital Communications at the University of Alberta, Canada. He is the author of the research monographs "Trellis

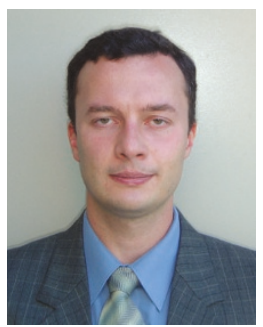
Coding" and "Trellis and Turbo Coding" by IEEE/Wiley, as well as "Coordinated Multiple User Communications," coauthored with Professor Alex Grant, published by Springer. Dr. Schlegel received an 1997 Career Award, and a Canada Research Chair in 2001. Dr. Schlegel is an Associate Editor for coding theory and techniques for the IEEE transactions on communications, and a Guest Editor of the IEEE proceedings on turbo coding. He served as the technical program Cochair of ITW 2001 and ISIT'05. He was also the general Chair of the CTW '05, as well as member of numerous technical program committees. 